\title{
FLAT QUASI-COHERENT SHEAVES OF FINITE COTORSION DIMENSION
}

\author{
ESMAEIL HOSSEINI
}

\begin{abstract}
Let $X$ be e quasi-compact and semi-separated scheme. If every flat quasicoherent sheaf has finite cotorsion dimension, we prove that $X$ is $n$-perfect for some $n \geq 0$. If $X$ is coherent and $n$-perfect(not necessarily of finite krull dimension), we prove that every flat quasi-coherent sheaf has finite pure injective dimension. Also, we show that there is an equivalence $\mathbf{K}(\operatorname{Pinf} X) \longrightarrow \mathbf{D}$ (Flat $X)$ of homotopy categories, whenever $\mathbf{K}(\operatorname{Pinf} X)$ is the homotopy category of pure injective flat quasi-coherent sheaves and $\mathbf{D}($ Flat $X)$ is the pure derived category of flat quasi-coherent sheaves.
\end{abstract}

\section{INTRODUCTION}

In this paper, $X$ denotes a quasi-compact and semi-separated scheme, $\mathcal{O}_{X}$-modules are quasi-coherent sheaves on $X$ and all rings are commutative with identity.

Let $\mathfrak{Q} \mathfrak{c o} X$ be the category of $\mathcal{O}_{X}$-modules. An $\mathcal{O}_{X}$-module $\mathcal{F}$ is called flat if for each $p \in X, \mathcal{F}_{p}$ is a flat $\mathcal{O}_{X, p}$-module or equivalently the the functor $\mathcal{F} \otimes_{\mathcal{O}_{X}}-: \mathfrak{Q} \mathfrak{c o} X \longrightarrow$ $\mathfrak{Q} \mathfrak{c o} X$ is exact. In [EE, 4.2], the authors proved that the pair (FlatX, CotX) is a complete cotorsion theory in $\mathfrak{Q} \mathfrak{c o} X$, whenever FlatX is the class of all flat $\mathcal{O}_{X}$-modules and $\operatorname{CotX}=\left\{\mathcal{G} \in \mathfrak{Q} \mathfrak{c o} X: \forall \mathcal{F} \in\right.$ FlatX, $\left.\operatorname{Ext}_{X}^{1}(\mathcal{F}, \mathcal{G})=0\right\}$, is the class of all cotorsion $\mathcal{O}_{X^{-}}$ modules. So, for a given $\mathcal{O}_{X}$-module $\mathcal{G}$, we can define $\operatorname{cd} \mathcal{G}$ (the cotorsion dimension of $\mathcal{G})$.

In this paper we study those schemes $X$ such that over them every flat $\mathcal{O}_{X}$-module has finite cotorsion dimension. If $X$ is affine, then every flat $\mathcal{O}_{X}$-module has finite projective dimension and so there exist an integer $n \geq 0$ such that for each flat $\mathcal{O}_{X^{-}}$ module $\mathcal{F}, \operatorname{pd} \mathcal{F} \leq n$ (the projective dimension of $\mathcal{F})$. Unfortunately, this argument is not true when $X$ is non-affine, since there is no non-zero projective $\mathcal{O}_{X}$-modules. In the following result we make a proof to such case.

Theorem 1.1. The following conditions are equivalent:

(i) Every flat $\mathcal{O}_{X}$-module has finite cotorsion dimension.

(ii) $X$ is $n$-perfect for some integer $n \geq 0$.

In the remainder of this paper we show that every flat $\mathcal{O}_{X}$-module has finite pure injective dimension. As an application, we prove that if $X$ is coherent $n$-perfect then there is an equivalence $\mathbf{K}(\operatorname{Pinf} X) \longrightarrow \mathbf{K}_{\mathrm{p}}($ Flat $X)$ of homotopy categories, whenever $\mathbf{K}($ Flat $X)$ be the homotopy category of complexes of flat $\mathcal{O}_{X}$-modules and $\mathbf{K}(\operatorname{Pinf} X)$ be the essential image of the homotopy category of complexes of pure injective flat $\mathcal{O}_{X}$-modules in $\mathbf{K}($ Flat $X)$ in the sense of [HS].

\footnotetext{
Key words and phrases. Quasi-coherent sheaf, cotorsion dimension, n-perfect scheme, homotopy category.

2010 Mathematical subject classification: 14F05, 18G20, 18E30.
} 
Setup. In this paper, $\mathfrak{U}=\left\{\operatorname{Spec} A_{i}\right\}_{i=1}^{m}$ denotes a semi-separating cover of $X$ (i.e. each intersection of elements of $\mathfrak{U}$ is also affine).

\section{Cotorsion Envelope OF BOUnded COMPlEXES}

Let $\mathbf{C}^{\mathrm{b}}(\mathfrak{Q} \mathfrak{c o} X)$ be the category of bounded complexes of $\mathcal{O}_{X}$-modules, $\mathbf{C}_{\mathrm{ac}}^{\mathrm{b}}($ FlatX) be the category of bounded acyclic complexes of flat $\mathcal{O}_{X}$-modules and $\mathbf{C}^{\mathrm{b}}(\operatorname{Cot} X)$ be the category of bounded complexes of cotorsion $\mathcal{O}_{X}$-modules. In this section, we prove that the pair $\left(\mathbf{C}_{\mathrm{ac}}^{\mathrm{b}}(\right.$ FlatX $\left.), \mathbf{C}^{\mathrm{b}}(\operatorname{Cot} X)\right)$ is a complete cotorsion theory in $\mathbf{C}^{\mathrm{b}}(\mathfrak{Q} \mathfrak{c o} X)$, i.e. $\mathbf{C}_{\mathrm{ac}}^{\mathrm{b}}($ FlatX $)={ }^{\perp} \mathbf{C}^{\mathrm{b}}(\operatorname{Cot} X), \mathbf{C}_{\mathrm{ac}}^{\mathrm{b}}(\text { FlatX })^{\perp}=\mathbf{C}^{\mathrm{b}}(\operatorname{Cot} X)$ and it has enough projectives. For notations and definitions see $[\mathrm{HS}]$ and $[\mathrm{EJ}]$.

Lemma 2.1. Let $\mathbf{G}: 0 \longrightarrow \mathcal{G}^{\prime} \longrightarrow \mathcal{G} \longrightarrow \mathcal{G}^{\prime \prime} \longrightarrow 0$ be an exact sequence of $\mathcal{O}_{X}$-modules. Then there exists a morphism $\phi: \mathbf{F} \longrightarrow \mathbf{G}$ of complexes whenever $\mathbf{F}$ is a short exact sequence of flat $\mathcal{O}_{X}$-modules.

Proof. Let $0 \longrightarrow \mathcal{C}^{\prime \prime} \longrightarrow \mathcal{F}^{\prime \prime} \longrightarrow \mathcal{G}^{\prime \prime} \longrightarrow 0$ be the flat cover of $\mathcal{G}^{\prime \prime}$. Consider the pullback diagram

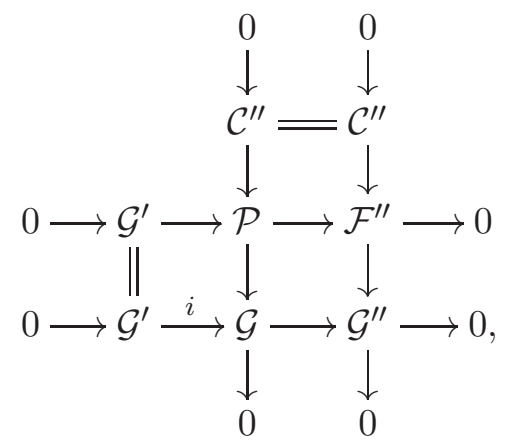

and let $0 \longrightarrow \mathcal{C}^{\prime} \longrightarrow \mathcal{F} \stackrel{p}{\longrightarrow} \mathcal{P} \longrightarrow 0$ be the flat cover of $\mathcal{P}$. Then the pullback of $i$ and $p$ completes the proof.

Recall that, a bounded complex $\mathbf{F}$ is called flat if $\mathbf{F} \in \mathbf{C}_{\mathrm{ac}}^{\mathrm{b}}$ (FlatX) and a bounded complex $\mathbf{C}$ is called cotorsion if $\mathbf{C} \in \mathbf{C}_{\mathrm{ac}}^{\mathrm{b}}(\text { FlatX })^{\perp}$, where the orthogonal is taken in the exact category $\mathbf{C}^{\mathrm{b}}(\mathfrak{Q} \mathfrak{c o} X)$. By similar argument that used in [HS, Proposition 2.1], we deduce the following proposition.

Proposition 2.2. Let $\mathbf{C}$ be a bounded complex. Then $\mathbf{C}$ is cotorsion if and only if it is a complex of cotorsion $\mathcal{O}_{X}$-modules.

Theorem 2.3. Let $\mathbf{X}$ be a bounded complex of $\mathcal{O}_{X}$-modules. Then there exists an exact sequence $0 \longrightarrow \mathbf{C} \longrightarrow \mathbf{F} \longrightarrow \mathbf{X} \longrightarrow 0$ of complexes, where $\mathbf{F}$ is flat and $\mathbf{C}$ is cotorsion.

Proof. By [Sp], there exists a quasi-isomorphism $f: \mathbf{X}[-1] \longrightarrow \mathbf{I}$, with $\mathbf{I}$ is a bounded complex of injective $\mathcal{O}_{X}$-modules. By Lemma 2.1, we construct the short exact sequence $0 \longrightarrow \mathbf{C}^{\prime} \longrightarrow \mathbf{F} \longrightarrow$ cone $(f) \longrightarrow 0$, with $\mathbf{F}$ is flat and $\mathbf{C}^{\prime}$ is cotorsion complex. Then the pullback of the morphisms $\mathbf{F} \longrightarrow \operatorname{cone}(f)$ and $\mathbf{I} \longrightarrow \operatorname{cone}(f)$ completes the proof.

Theorem 2.4. The pair $\left(\mathbf{C}_{\mathrm{ac}}^{\mathrm{b}}(\mathrm{FlatX}), \mathbf{C}^{\mathrm{b}}(\operatorname{Cot} X)\right)$ is a complete cotorsion theory in $\mathrm{C}^{\mathrm{b}}(\mathfrak{Q} \mathfrak{c o} X)$. 
Proof. It suffices to show that $\mathbf{C}_{\mathrm{ac}}^{\mathrm{b}}($ FlatX $)={ }^{\perp} \mathbf{C}^{\mathrm{b}}(\operatorname{Cot} X)$. Let $\mathbf{X} \in{ }^{\perp} \mathbf{C}^{\mathrm{b}}(\operatorname{Cot} X)$. By Theorem 2.3, there exist an exact sequence $0 \longrightarrow \mathbf{C} \longrightarrow \mathbf{F} \longrightarrow \mathbf{X} \longrightarrow 0$, of complexes, with $\mathbf{F}$ is flat and $\mathbf{C}$ is cotorsion. By assumption this is split exact sequence. Then $\mathbf{X} \in \mathbf{C}_{\mathrm{ac}}^{\mathrm{b}}\left(\right.$ FlatX). Therefore $\left(\mathbf{C}_{\mathrm{ac}}^{\mathrm{b}}(\right.$ FlatX $\left.), \mathbf{C}^{\mathrm{b}}(\operatorname{Cot} X)\right)$ is a cotorsion theory which is complete by Theorem 2.3 .

Corollary 2.5. Every bounded complex of $\mathcal{O}_{X}$-modules admits flat cover and cotorsion envelope.

\section{COTORSION DIMENSION OF FLAT $\mathcal{O}_{X}$-MODULE}

In this section we prove that if every flat $\mathcal{O}_{X}$-module has finite cotorsion dimension then $X$ is $n$-perfect for some $n \geq 0$.

Definition 3.1. Let $n \geq 0$ be an integer. $X$ is called $n$-perfect if $n=\sup \{\operatorname{cd} \mathcal{F} \mid \mathcal{F} \in$ Flat $X\}$.

Theorem 3.2. Let for each $1 \leq i \leq m$, every flat $A_{i}$-module has finite cotorsion dimension. Then $X$ is n-perfect for some $n$.

Proof. Let $\mathcal{F}$ be a flat $\mathcal{O}_{X}$-module and

$$
\mathbf{G}: 0 \longrightarrow \mathcal{F} \longrightarrow \mathfrak{C}^{0}(\mathfrak{U}, \mathcal{F}) \longrightarrow \mathfrak{C}^{1}(\mathfrak{U}, \mathcal{F}) \longrightarrow \cdots \longrightarrow \mathfrak{C}^{m-2}(\mathfrak{U}, \mathcal{F}) \longrightarrow \mathfrak{C}^{m-1}(\mathfrak{U}, \mathcal{F}) \longrightarrow 0
$$

be its Čheck resolution. By assumption, for each $0 \leq i \leq m-1, \mathfrak{C}^{i}(\mathfrak{U}, \mathcal{F})$ has finite cotorsion dimension, then by Theorem 2.4 there exist a resolution

$$
0 \longrightarrow \mathbf{G} \longrightarrow \mathbf{C}_{0} \longrightarrow \mathbf{C}_{1} \longrightarrow \cdots \longrightarrow \mathbf{C}_{n-1} \longrightarrow \mathbf{C}_{n} \longrightarrow 0
$$

of $\mathbf{G}$ by flat complexes of $\mathcal{O}_{X}$-modules, where $\mathbf{C}_{0}, \mathbf{C}_{1}, \ldots, \mathbf{C}_{n-1}$ are cotorsion complexes and $\mathbf{C}_{n}$ is a flat complex such that for each $i>0, \mathbf{C}_{n}^{i}$ is cotorsion. Then the flat complex

$$
0 \longrightarrow \mathcal{F} \longrightarrow \mathbf{C}_{0}^{0} \longrightarrow \mathbf{C}_{1}^{0} \longrightarrow \cdots \longrightarrow \mathbf{C}_{n-1}^{0} \longrightarrow \mathbf{C}_{n}^{1} \longrightarrow \mathbf{C}_{n}^{2} \longrightarrow \cdots \longrightarrow \mathbf{C}_{n}^{m} \longrightarrow 0
$$

is a cotorsion resolution of $\mathcal{F}$.

Theorem 3.3. If every flat $\mathcal{O}_{X}$-module has finite cotorsion dimension. Then for each $1 \leq i \leq m$, every flat $A_{i}$-module has finite cotorsion dimension.

Proof. With out lose of generality we can assume that $i=1$. Let $F$ be a flat $A_{1}$ module, $f: U_{1} \longrightarrow X$ be the inclusion and $\mathbf{C}_{F}: 0 \longrightarrow F \stackrel{\xi}{\longrightarrow} C^{0} \stackrel{\delta^{0}}{\longrightarrow} C^{1} \stackrel{\delta^{1}}{\longrightarrow} C^{2} \stackrel{\delta^{2}}{\longrightarrow} \cdots$, be its minimal cotorsion resolution. By construction, $\mathbf{C}_{F}$ is a pure acyclic complex of flat $A_{1}$-modules. Apply the exact functor $f_{*}$ and get the pure acyclic complex

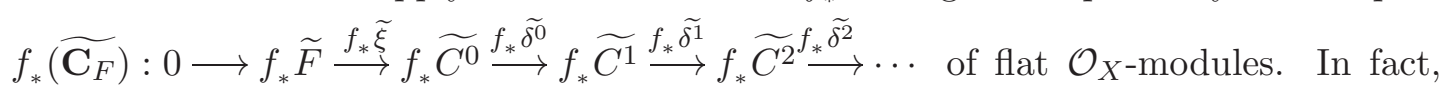
it is a cotorsion resolution of $f_{*} \widetilde{F}$. The assumption implies that $\operatorname{Im} f_{*} \widetilde{\delta^{n-1}}$ is cotorsion for some integer $n$. So the exact sequence

$$
0 \longrightarrow \operatorname{Im} f_{*} \widetilde{\delta^{n-1}} \longrightarrow f_{*}\left(\widetilde{C^{n}}\right) \longrightarrow \operatorname{Im} f_{*} \widetilde{\delta^{n}} \longrightarrow 0
$$

of flat $\mathcal{O}_{X}$-modules splits. Then $C^{n}=\operatorname{Im} \delta^{n-1} \oplus \operatorname{Im} \delta^{n}$. It follows that $\operatorname{cd} F \leq n$. 
Proof of Theorem 1.1. $(i) \longrightarrow$ (ii) If every flat $\mathcal{O}_{X}$-module has finite cotorsion dimension. Then by Theorem 3.3, for each $0 \leq i \leq m$, every flat $A_{i}$-module has finite cotorsion dimension. So, for each $i$ there exist an integer $n_{i} \geq 0$ such that $A_{i}$ is $n_{i^{-}}$ perfect. Therefore the proof of Theorem 3.2 implies that $X$ is $n$-perfect for some integer $n \geq 0$.

$($ ii $) \longrightarrow($ i) Clear.

Theorem 3.4. A scheme $X$ is n-perfect if and only if for every $\mathcal{O}_{X}$-module $\mathcal{G}$, cd $\mathcal{G} \leq n$.

Proof. Let $X$ be $n$-perfect, $\mathcal{G}$ be an $\mathcal{O}_{X}$-module and $0 \longrightarrow \mathcal{C} \longrightarrow \mathcal{F}^{\prime} \longrightarrow \mathcal{G} \longrightarrow 0$ be the flat cover of $\mathcal{G}$. Then for any flat $\mathcal{O}_{X}$-module $\mathcal{F}$ we have the following exact sequence

$$
0=\operatorname{Ext}_{X}^{n+1}(\mathcal{F}, \mathcal{C}) \longrightarrow \operatorname{Ext}_{X}^{n+1}\left(\mathcal{F}, \mathcal{F}^{\prime}\right) \longrightarrow \operatorname{Ext}_{X}^{n+1}(\mathcal{F}, \mathcal{G}) \longrightarrow \operatorname{Ext}_{X}^{n+2}(\mathcal{F}, \mathcal{C})=0 .
$$

Then $\operatorname{Ext}_{X}^{n+1}(\mathcal{F}, \mathcal{G})=0$ and hence $\operatorname{cd} \mathcal{G} \leq n$.

The converse is trivial.

Now by using the main Theorem of [Si] we give examples of non-noetherian $n$-perfect schemes of infinite Krull dimension.

Example 3.5. Let $R$ be a ring, $|R| \leq \aleph_{n}$ for some $n \geq 0, A=R\left[x_{1}, x_{2}, \ldots\right]$ be the polynomial ring of infinite indeterminate and $X=\bigcup_{i=1}^{i=m} D\left(f_{i}\right)$ be an open subscheme of $\operatorname{Spec} A$. Then $X$ is a non-noetherian non-affine scheme of infinite krull dimension(for definitions and notations see [H, II.2]). By the same argument that used in the proof of Theorem 3.2 we deduce that $X$ is $k$-perfect for some $k$.

Example 3.6. Let $\mathfrak{T}$ be a topological space of cardinality at most $\aleph_{n}$ for some integer $n \geq 0$. If $\mathfrak{T}$ is not $p$-space, then the commutative $\operatorname{ring} \mathrm{C}(\mathfrak{T})$, the ring of real valued continuous functions on $\mathfrak{T}$, is a non-noetherian $(n+1)$-perfect ring of infinite krull dimension. For example the metric space $\mathbb{R}$ (real numbers) is not a $p$-space.

Example 3.7. If $R$ is a noetherian ring of finite krull dimension $n$. Then it is $n$-perfect.

Example 3.8. If $R$ is $n$-perfect. Then $R[x]$ is also $(n+1)$-perfect.

Example 3.9. The Nagata's example of a noetherian ring of infinite krull dimension is $n$-perfect for some integer $n$, see[Appendix, Example 1][Na]

3.1. Pure injective dimension of flat $\mathcal{O}_{X}$-modules. Recall that an exact sequence $0 \longrightarrow \mathcal{K} \longrightarrow \mathcal{G}$ of $\mathcal{O}_{X}$-modules is called pure if it remains exact after tensoring with any $\mathcal{O}_{X}$-module. An $\mathcal{O}_{X}$-module $\mathcal{E}$ is called pure injective if it is injective with respect pure exact sequences of $\mathcal{O}_{X}$-modules. For a given $\mathcal{O}_{X}$-module $\mathcal{F}$, let $\mathcal{F}^{*}=\oplus_{i=1}^{m} f_{i_{*}} \widetilde{F_{i}^{*}}$, $\mathcal{F}^{* *}=\oplus_{i=1}^{m} f_{i *} \widetilde{F_{i}^{* *}}$ such that for each $1 \leq i \leq m, F_{i}=\mathcal{F}\left(U_{i}\right), F_{i}^{*}=\operatorname{Hom}_{\mathbb{Z}}\left(F_{i}, \mathbb{Q} / \mathbb{Z}\right)$, and $f_{i}: U_{i} \longrightarrow X$ be the inclusion. Then $\mathcal{F}^{*}$ and $\mathcal{F}^{* *}$ are pure injective $\mathcal{O}_{X}$-modules and $\mathcal{F} \longrightarrow \mathcal{F}^{* *}$ is a pure monomorphism.

In this subsection we let $X$ be a coherent scheme. Recall that $X$ is called coherent if $A_{i}$ is a coherent ring for each $1 \leq i \leq m$

Proposition 3.1.1. Let $\mathcal{F}$ be a flat $\mathcal{O}_{X}$-module. Then $\mathcal{F}$ is pure injective if and only if it is cotorsion. 
Proof. Let $0 \longrightarrow \mathcal{F} \longrightarrow \mathcal{C} \longrightarrow \mathcal{G} \longrightarrow 0$ be the cotorsion envelope of $\mathcal{F}$. Since $\mathcal{F}$ is flat then this sequence is pure and hence it is split.

Let $\mathcal{F}$ be a cotorsion $\mathcal{O}_{X}$-module and $0 \longrightarrow \mathcal{F} \longrightarrow \mathcal{F}^{* *} \longrightarrow \frac{\mathcal{F}^{* *}}{\mathcal{F}} \longrightarrow 0$ be its pure injective preenvelope. Since $\mathcal{F}$ and $\mathcal{F}^{* *}$ are flat $\mathcal{O}_{X}$-module then $\frac{\mathcal{F}^{* *}}{\mathcal{F}}$ is also flat and so this sequence is split.

The pure injective dimension of an $\mathcal{O}_{X}$-module $\mathcal{F}$ can be defined in usual sense.

Theorem 3.1.2. A scheme $X$ is n-perfect if and only if every $\mathcal{O}_{X}$-module has finite pure injective dimension.

Proof. Let $\mathcal{G}$ be an $\mathcal{O}_{X}$-module and

$$
0 \longrightarrow \mathcal{G} \longrightarrow \mathcal{C}^{0} \stackrel{\delta^{0}}{\longrightarrow} \mathcal{C}^{1} \stackrel{\delta^{1}}{\longrightarrow} \cdots
$$

be its minimal cotorsion resolution. By Theorem 3.4, $\operatorname{Im} \delta^{n-1}$ is cotorsion flat and by Proposition 3.1.1, it is pure injective. Therefore this pure exact sequence is a pure injective resolution of $\mathcal{G}$ of length $n$.

By Proposition 3.1.1, the converse is trivial.

\section{Applichtion}

Let $\mathbf{K}($ Flat $X)$ be the homotopy category of complexes of flat $\mathcal{O}_{X}$-modules, $\mathbf{K}_{\mathrm{p}}($ Flat $X)$ be the full subcategory of $\mathbf{K}($ Flat $X)$ consisting of all pure acyclic complexes of flat $\mathcal{O}_{X^{-}}$ modules and $\mathbf{K}(\operatorname{Cof} X)$ be the essential image of the homotopy category of complexes of cotorsion flat $\mathcal{O}_{X}$-modules. In [HS], the authors proved that there is an equivalence $\mathbf{K}(\operatorname{Cof} X) \longrightarrow \mathbf{K}($ Flat $X) / \mathbf{K}_{\mathrm{p}}($ Flat $X)=\mathbf{D}($ Flat $X)$ of homotopy categories, whenever $\mathbf{K}_{\mathrm{p}}($ Flat $X) \cap \mathbf{K}(\operatorname{Cof} X)=0$ and $\mathfrak{Q} \mathfrak{c o} X$ have enough flats. For instance such equivalenece of homotopy categories exists, when $X$ is $n$-perfect (possibly non-noetherian of infinite Krull dimension).

In this section we let $X$ be a coherent, $\mathbf{C}_{\mathrm{p}}($ Flat $X)$ be the category of all flat complexes of $\mathcal{O}_{X}$-modules and $\mathbf{C}(\operatorname{Pinj} X)$ be the category of complexes of pure injective $\mathcal{O}_{X}$-modules.

Theorem 4.1. Let $\mathbf{C}$ be a complex of $\mathcal{O}_{X}$-modules. Then $\mathbf{C} \in \mathbf{C}_{\mathrm{p}}(\text { Flat } X)^{\perp}$ if and only if it is a complex of pure injective $\mathcal{O}_{X}$-modules .

Proof. Let $\mathbf{C}$ be a complex of pure injective $\mathcal{O}_{X}$-modules. By [HS, Proposition 2.6], there is a degree-wise split exact sequence $0 \longrightarrow \mathbf{C} \longrightarrow \mathbf{C}^{\prime} \longrightarrow \mathbf{F}^{\prime} \longrightarrow 0$ where $\mathbf{C}^{\prime} \in$ $\mathbf{C}_{\mathrm{p}}(\text { Flat } X)^{\perp}$ and $\mathbf{F}^{\prime} \in \mathbf{C}_{\mathrm{p}}($ Flat $X)$. Therefore we have a canonical morphism $u: \mathbf{F}^{\prime} \rightarrow$ $\Sigma \mathbf{C}$ such that $\mathbf{C} \longrightarrow \mathbf{C}^{\prime} \longrightarrow \mathbf{F}^{\prime} \stackrel{u}{\longrightarrow} \Sigma \mathbf{C}$ is a triangle in $\mathbf{K}($ Flat $X)$. Moreover, $\mathbf{F}^{\prime}$ is a complex of cotorsion $\mathcal{O}_{X}$-modules and hence it is contractible by $n$-perfectness of $X$. It follows that for each flat complex $\mathbf{F}, \operatorname{Hom}_{\mathbf{K}(X)}(\mathbf{F}, \mathbf{C}) \cong \operatorname{Hom}_{\mathbf{K}(X)}\left(\mathbf{F}, \mathbf{C}^{\prime}\right)=0$. Therefore by [HS, Proposition 2.1], $\mathbf{C} \in \mathbf{C}_{\mathrm{p}}(\text { Flat } X)^{\perp}$.

The converse follows from [HS, Proposition 2.1].

Corollary 4.2. The cotorsion theory $\left(\mathbf{C}_{\mathrm{p}}(\right.$ Flat $\left.X), \mathbf{C}(\operatorname{Pinj} X)\right)$ is complete.

Proof. The result follows from [HS, Theorem 2.5] and Theorem 4.1. 
Let $\mathbf{K}(\operatorname{Pinf} X)$ be the essential image(in the sense of [HS]) of the homotopy category of complexes of pure injective flat $\mathcal{O}_{X}$-modules in $\mathbf{K}($ Flat $X)$.

Corollary 4.3. There is an equivalence $\mathbf{K}(\operatorname{Pinf} X) \longrightarrow \mathbf{D}($ Flat $X)$ of homotopy categories.

Proof. The pair $\left(\mathbf{K}_{\mathrm{p}}(\right.$ Flat $\left.X), \mathbf{K}(\operatorname{Pinf} X)\right)$ is a complete cotorsion theory in $\mathbf{K}($ Flat $X)$ in the sense of $[\mathrm{HS}]$. Then there is an equivalence $\mathbf{K}(\operatorname{Pinf} X) \longrightarrow \mathbf{D}($ Flat $X)$ of homotopy categories.

\section{REFERENCES}

[EE] E. Enochs, S. EstradA, Relative homological algebra in the category of quasi-coherent sheaves , Adv.Math. 194 (2005), 284-295.

[EJ] E. Enochs , O. Jenda, Relative homological algebra, Gordon and Breach S.Publishers, (2000).

[H] R. Hartshorne, Algebraic Geometry, Springer- Verlag, New York Inc. (1997).

[HS] E. Hosseini, Sh. Salarian, A cotorsion theory in the homotopy category of flat quasi-coherent sheaves, Proc. Amer. Math. Soc. 141 (3) (2013), 753-762.

[Na] M. Nagata, Local rings, R. E. Krieger Pub. Co. 234 (1975).

[Si] D. Simson, A remark on projective dimension of flat modules, Math. Ann. 209 (1974), 181182.

[Sp] N. Spaltenstein, Resolutions of unbounded complexes, Compositio Math. 65, no. 2 (1988), 121-154.

Department of Mathematics, Shahid Chamran University of Ahvaz, Ahvaz, Iran

E-mail address: esmaeilmath@gmail.com 